\title{
Cardiac Outpatient Care in a Digital Age: Remote Cardiology Clinic Visits in the Era of COVID-19
}

\author{
Kunal Mishra ${ }^{1}$ [D $\cdot$ Brian Edwards ${ }^{1}$ \\ Accepted: 15 September 2021 / Published online: 14 January 2022 \\ (c) The Author(s), under exclusive licence to Springer Science+Business Media, LLC, part of Springer Nature 2022
}

\begin{abstract}
Purpose of Review The worldwide pandemic caused by the novel coronavirus disease transformed healthcare in many ways. The impact of the pandemic was also noted in outpatient settings with various clinics adopting telehealth as the new normal. The goal of this paper is to investigate how the pandemic impacted the outpatient cardiology setting, specifically regarding the use of telehealth, and can the lessons learned from the adoption of telehealth in the backdrop of COVID-19 be applied to facilitate the wider and routine use of telemedicine in the outpatient cardiology clinic.

Recent Findings Several studies have been conducted showcasing COVID-19's impact on the telehealth field of cardiology. Studies showed advantages for patients. Among these advantages are reduction in wait and travel time, easier medication reconciliation, and convenience. They also showed a general comfortability with the transition to telehealth among cardiologists. Furthermore, the adoption of telehealth in the outpatient cardiology setting, specifically with respect to the management of common cardiac conditions of congestive heart failure, atrial fibrillation, and ischemic heart disease, revealed the potential of telemedicine to be used to adequately address these conditions. The transition to telehealth was not without its challenges, such as lack of a physical exam, barriers with certain patient populations to adopting the technology, and changes were noted in frequencies of medication ordering and cardiology-specific laboratory and diagnostic imaging.

Summary This transition to telehealth during the pandemic allowed for various studies to be conducted on how telehealth impacted the field of cardiology in the outpatient setting. While patient and practitioner advantages were revealed when compared to traditional outpatient cardiology visits, barriers to the adoption of the technology among specific patient populations were noted as were changes in practice among cardiologists. The use of telemedicine to adequately address common cardiac conditions was also shown. Further investigation into understanding the barriers of specific patient populations and overcoming these barriers, understanding the reason for the changes in practice of cardiologists with the use telemedicine, and investigating the use and incorporation of existing technology such as smart watches and patient portals or apps to make the transition to telehealth not only simpler, but to also optimize the cardiologist management of common cardiac conditions, have the potential to lead to the wider and routine use of telemedicine in the outpatient cardiology clinic.
\end{abstract}

Keywords COVID-19 pandemic $\cdot$ Cardiology $\cdot$ Cardiac outpatient visits $\cdot$ Telehealth

\section{Introduction}

The novel coronavirus disease (COVID-19) was declared to be a global pandemic on March 12, 2020, by the World Health Organization [1]. In an effort to try to mitigate the

This article is part of the Topical Collection on Ischemic Heart Disease

Kunal Mishra

kunal.mishra@ttuhsc.edu

1 Department of Internal Medicine, Texas Tech University Health Sciences Center, MSC 41007, 4801 Alberta Avenue, El Paso, TX 79905, USA transmission of this deadly disease, several recommendations were enacted by the CDC, among them were encouraging the populace to limit any outdoor activities, including elective in-person clinic visits [2]. These recommendations had a significant impact with regard to ambulatory care. For decades, many restrictions had prevented expansion of telehealth services, however, on March 17, 2020, the Centers for Medicare and Medicaid Services (CMS) issued an 1135 waiver which allowed for the expansion of telehealth coverage for all Medicare patients. This allowed physicians to expand their use of telemedicine by eliminating barriers such as lack of reimbursement, licensing restrictions, and HIPAA compliance [3]. The pandemic along with removal 
of barriers to telehealth expansion caused a shift from in person ambulatory care to remote visits as a way to deliver medical care without risking transmission [2].

Telehealth offered an easy way for physicians to provide care and maintain connections with their patients, all while practicing social distancing [3]. This shift to telemedicine was not just limited to primary care. Several specialties adopted telehealth as a way to deliver care. A study conducted at the University of California Comprehensive Cancer Center showed that a shift to telehealth visits allowed patients to maintain access to complex oncologic care without any disparity based on race/ ethnicity, primary language, or insurance, during the pandemic [4]. The American College of Obstetrics \& Gynecology had also released guidelines on the use of telehealth during the pandemic. This allowed for several obstetrics \& gynecology practices to shift their ambulatory care to telehealth comfortably [5]. Another study showed great patient satisfaction with telehealth for both gastroenterology providers and patients when the use of telehealth increased dramatically during the COVID-19 pandemic [6].

The field of cardiology presented a unique opportunity for telehealth, as cardiovascular disease (CVD) commonly occurs as a result of a combination of risk factors that must be continually addressed. The 2010 Global Burden of Disease study demonstrated that the burden of ischemic heart disease has risen significantly since 1990 with a significant proportion of the burden of CVD being preventable [7]. A 2010 study showed that in the US alone between $36 \%$ and $63 \%$ of myocardial infarctions could be prevented through risk factor reduction [8]. Continually treating these modifiable risk factors in outpatient visits is integral to preventing further CVD. Telehealth provided the perfect opportunity to deliver this care during the pandemic.

This paper seeks to investigate the advantages and potential barriers in regards to the use of telehealth in the field of cardiology. It will further investigate advantages, barriers and issues specific to certain diagnoses, and how telehealth has an impact on the practice of cardiologists in the outpatient setting.

\section{Cardiology and COVID-19}

As more and more studies on COVID-19 were published and the disease itself was studied, it became clear that common comorbidities such as hypertension, diabetes, and established cardiovascular disease conferred the highest risk of developing severe forms of COVID-19. In fact, the CDC lists the presence of heart failure, coronary artery disease, and hypertension as conditions that may put patients at a higher risk to become severely ill from COVID-19 [9, 10]. Furthermore, the presence of underlying CVD increases both the severity of the primary respiratory disease and the risk of developing subsequent complications [10,11]. A recent study found that CVD and associated risk factors were associated with fatal outcomes with COVID-19 across all age groups, thus demonstrating the importance of paying special attention to addressing cardiovascular comorbidities in the young and the elderly in outpatient visits [11]. However, with stay-at-home orders being given worldwide to reduce the transmission of COVID-19, outpatient visits to followup on these risk factors have also been reduced. The medical community responded to this change with the fast rise and expansion of telemedicine with the field of cardiology being no exception. With the adoption of telemedicine in cardiology, a set of new challenges and opportunities arose that very well may change the future of outpatient cardiology visits.

\section{Advantages to Telehealth in the Field of Cardiology}

With the transition to telemedicine, several advantages were noted in remote cardiology clinic visits. The most noted advantage was that patients saw a reduction in travel time and wait time. Patients also ranked reduced travel time as a significant advantage in addition to decreased travel costs [13••]. Patients also noted that with telehealth visits, there was a decreased wait time. Telehealth allowed for patients to be notified by telephone, thus avoiding the wait that sometimes accompanies in-person visits. Surveys of patients also show an increased comfort level with telemedicine visits and the ability to continue to work at

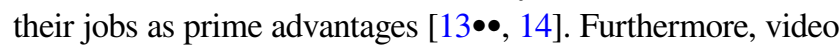
visits allow patients to show physicians their prescription bottles allowing for medication reconciliation to be done. Overall, patient satisfaction remained stable when comparing inperson visits and telehealth visits during the pandemic [13••, 14]. Additionally, there were studies showing that remote visits equally replaced the decrease in in-person patient visits during the COVID-era period, demonstrating patient comfort with the transition [15]. Cardiologists were also surveyed, and despite many having no prior experience, they were satisfied with the transition overall [16•]. With CMS having extended reimbursement for virtual visits during the COVID-era, time will tell if these changes are here to stay. However, with what appears to be the successful transition to telehealth in cardiology clinics, it would behoove CMS to continue to extend this and further grow this advancement.

\section{Potential Barriers to Telehealth in Cardiology}

The great potential of telehealth has always been tempered by concerns of lack of access. Patients who are older and who live in rural areas with lack of consistent internet or have less 
education may be at higher risk of not receiving telehealth $[13 \bullet \bullet, 17]$. Additionally, communities that are underserved with healthcare services may also be those with limited access to reliable internet service or lack understanding on how to deploy telehealth technology [17]. Studies that focused on cardiology outpatient telehealth visits revealed a sharp contrast from this theory. These studies revealed that during the pandemic, internet and technology access were not significant barriers to the use of telehealth [13••]. A study conducted in Kentucky found that among their respondents who declined to use telehealth, only $6.2 \%$ cited access to technology as a reason [6]. Furthermore, more than $70 \%$ of respondents reported unfamiliarity with technology as not being a factor in declining telehealth or as a disadvantage, among those who participated. These findings suggest that even during a period with rapid change to telehealth, internet access or the use of technology did not act as significant barriers.

While access to technology seemingly did not act as a barrier, age appeared to be one such factor. Older patients had difficulties with telemedicine technology including using video visits less frequently [16•]. This is consistent with prior literature which has shown lower rates of telemedicine use among patients $75-84$ years old [18, 19]. This is rather concerning with respect to cardiology visits, as elderly patients tend to have multiple comorbidities and risk factors that require close monitoring. A cross-sectional study by Yuan et al. showed that older patients used video visits less frequently for cardiology remote visits. However, recent data has shown that a growing number of older adults have smartphones and that with some guidance on using new technology by clinic support staff or the integration of telehealth into a platform already familiar with patients, such as patient portals, older adults may be able to combat this disparity. As telehealth grows, different approaches to combat the barriers that the elderly face will be necessary [16•].

In addition to age, the pandemic revealed inequities in care which may be manifested with the use of telemedicine. Populations that were non-English speaking, poorer, lacked insurance, female, and as previously mentioned, older, were associated with a lower telemedicine use. The inability to speak English was found to be independently associated with a $>50 \%$ lower telemedicine use [12]. While virtual interpreter services do exist, the adoption of such services into telehealth must occur to address this inequity. With the shift to telemedicine, studies have shown a modest decline in outpatient visits in lower income populations with no consensus as to a possible etiology. Despite the seeming advantages of telemedicine, healthcare disparities are not eliminated with its use. As telemedicine continues to grow, attention to disparities in the aforementioned populations, as well as barriers which may not have revealed themselves given the pioneering and somewhat experimental nature of telehealth expansion, needs to remain a focus.
A perceived barrier from the physician side of telehealth included the lack of a comprehensive physical exam [16•]. This issue is particularly important in the field of cardiology as the physical exam can influence the subsequent laboratory or imaging orders physicians may make. Findings such as an irregular rhythm or volume status, which can be easily checked with a comprehensive physical exam, can be difficult, if not impossible, to do in a telehealth visit. Basic components of the physical examination can be performed over video-assisted telehealth visits such as general appearance, alertness and orientation, and checking for signs of peripheral edema. Recent developments in video technology have also showed that magnification into the patient's neck veins may accurately correlate with invasive measured right atrial pressure $[15,16 \bullet]$. However, these benefits may all be null, as elderly populations struggle to use video visits properly. Furthermore, there is a decrease in patient satisfaction between in-person and telehealth visits regarding the clinical exam due to lack of video [16•]. Thus, in order for the use of telehealth to expand within the field of cardiology, mechanisms must be put in place that allow physicians and healthcare administrators to educate their patients on the usage of this technology to better facilitate video visits to allow for a basic physical exam.

\section{Specific Cardiology Diagnoses in the Outpatient Setting}

\section{Congestive Heart Failure}

Congestive heart failure (CHF) represents one aspect of cardiology that requires routine outpatient care, thus making it the perfect platform to see telehealth in action. Telemedicine visits allow physicians to perform medication reconciliation, adjust dosages of guideline-directed medical therapy, counsel patients regarding diet and medication compliance, and discuss symptoms that may be problematic such as orthopnea, paroxysmal nocturnal dyspnea, and edema. Additionally, telehealth has allowed for patient caregivers or family members to be involved in patient appointments at a time when visiting restrictions would make this interaction or involvement impossible [20]. While compensated CHF patients can be easily managed via telehealth, assessing patients with acute symptoms over telehealth becomes challenging. Like in-person visits, telehealth visits should be classified as urgent or routine, and the physician must triage to determine if certain telehealth patients must be seen inperson. Urgent classification may encompass visits regarding worsening CHF symptoms such as acute dyspnea, excessive fatigue particularly with exertion, or development of edema or rapid weight gain, among others. Dyspnea, in particular, must be managed with special care in cardiology telehealth 
visits given the importance of differentiating an acute $\mathrm{CHF}$ exacerbation from COVID-19 infection. While the challenges of a lack of a complete physical exam and education of the elderly remain, especially in heart failure patients, solutions such as remote monitoring and elderly education rise as viable solutions. In fact, studies that included either automated monitoring of signs and symptoms or automated physiologic monitoring showed a $27-40 \%$ reduction in CHF decompensation admissions and a reduction of all-cause mortality [21, 22]. A recent retrospective study comparing home telemedicine care with outpatient care showed that home telemedicine was associated with improved survival. The trend of reduced mortality was seen throughout followups, up to 60 months from the initial encounter. The study theorized that the reduced mortality in home telemedicine patients may reflect the improved patient CHF knowledge and self-care behaviors that are reinforced during these visits [23]. Given the success of managing compensated heart failure patients via telehealth and the high patient satisfaction seen in studies, many CHF programs across the country have already converted previously in-person visits into telehealth visits, demonstrating a possible transition to telehealth medicine for chronic CHF patients in the future [20].

\section{Atrial Fibrillation}

Atrial fibrillation may be especially well suited for telehealth medicine as these visits lend themselves to incorporating remote patient monitoring. Studies before the pandemic have shown the value in digital electrocardiograms in detecting abnormal rhythms [24, 25]. Holter monitors and other rhythm monitors can be mailed to patients in the event a physician is concerned about a patient's heart rate. Furthermore, with the field of wearable smart watches increasing, more and more devices can recognize arrhythmias. Studies are currently being conducted to find if there is a benefit of wearable devices use in healthcare and its impact [26]. During these telehealth visits, all cardiac imaging can be reviewed with patients, and physicians can also assess for symptoms associated with uncontrolled atrial fibrillation. If there are any changes required in dosing of rate control medications, remote patient monitoring will play a valuable role $[24,25]$. While there are findings associated with atrial fibrillation, such as exertional syncope or worsening palpitations, which may necessitate that a patient should be seen inperson, the feasibility of chronic management via telehealth remains a possibility for these patients in the future.

\section{Ischemic Heart Disease}

In addition to common cardiovascular conditions such as atrial fibrillation and CHF, telehealth has significant potential in treating ischemic heart disease. Patients discharged after an acute coronary syndrome should be followed up promptly to check on symptoms, treatment adherence, and optimization [25]. All of these can be addressed during telehealth visits. Telehealth visits would also allow cardiologists to check on status of worsening ischemic heart disease symptoms such as worsening angina or new onset heart failure symptoms. While reviewing blood pressure and heart rate logs with the patient, cardiologists would be able to successfully further optimize treatments [25, 27]. Telemedicine visits would also allow for the routine counseling cardiologists provide regarding lifestyle modifications to be delivered. While further studies need to be determined as to the effectiveness of telehealth interventions in reducing overall CVD risk, the Spanish Society of Cardiology recently came out with a guide on how to treat ischemic heart disease over telehealth visits, thus providing an example of the faith in delivering adequate care via telehealth $[25,27]$.

\section{The Impact of Telehealth on Cardiologists}

The transition to telemedicine in cardiology visits has also impacted physician practices. Prior literature had concerns about telemedicine visits in regard to the frequency of laboratory testing and imaging orders or medication prescriptions, as experts argued that the lack of physical exam during remote visits would lead to the overuse of unnecessary testing and the over-prescribing of medications [13••]. A study by Yuan et al. showed a different result however, as there was a stepwise reduction in the ordering frequency of both diagnostic tests and prescription medications when comparing pre-COVID and COVID-era telemedicine visits to in-person visits [13••]. While the study offers no direct reason for why this occurred, the authors suggested reduced access, as many of the tests that would be ordered, such as labs, would often be performed in a facility which patients did not wish to visit particularly in light of the COVID-19 pandemic. Other studies have noted similar results with telehealth visits being associated with fewer new medication prescriptions, electrocardiograms, echocardiograms, and stress tests ordered [28]. There may also be a tie to the previously discussed issues with physical examination as the lack of physical exam may lead to physicians not feeling comfortable ordering tests or prescribing medications if not cued by findings on physical exam. Support for this theory gained more merit from a study which showed that telephone visits led to less tests or medications ordered when compared with video visits. Despite the possible reasons for the lower frequency of testing during telehealth visits, further research is required to determine if this finding is due to a lack of indication, patient discomfort with performing the tests during a pandemic or cardiologists feeling relatively less comfortable in a telehealth setting [28]. 


\section{Conclusion: Future of COVID, Cardiology, and Telemedicine}

With the rollout of COVID-19 vaccinations and reduction of restrictions from the $\mathrm{CDC}$, the pandemic may be in its final stages. The pandemic caused by COVID-19 however leaves behind a lasting impact on the healthcare system [15, 29]. With the slow return of society to normal, clinicians must decide whether there is a future for telehealth visits, and they will use the lessons learned from the pandemic to decide. Studies have shown very high satisfaction among patients and practitioners with virtual visits during the pandemic $[13 \bullet \bullet, 16 \bullet]$. While there are barriers and inequities in care still present, the field of telemedicine is fast growing. Particular to cardiology, telemedicine visits to address chronic medical illnesses such as CHF and atrial fibrillation may increase in the future as both cardiologists and patients become more comfortable with this mode of healthcare. Concerns regarding physical exam and lack of objective data during these visits are slowly being addressed with remote monitoring technologies such as biosensing wearables and watches and Holter monitors. Further studies will need to be done to determine if telehealth visits can lead to greater adherence, decrease no-show rates, improve access to healthcare, and improve transition of care [29]. However, now that the gates of telemedicine have been opened by the pandemic, it will be interesting to see how it continues to grow in the field of cardiology.

\section{Compliance with Ethical Standards}

Conflict of Interest The authors declare that they have no conflict of interest.

Human and Animal Rights and Informed Consent This article does not contain any studies with human or animal subjects performed by any of the authors.

\section{References}

Papers of particular interest, published recently, have been highlighted as:

- Of importance

$\bullet$ Of major importance

1. Fisk M, Livingstone A, Pit SW. Telehealth in the context of COVID-19: changing perspectives in Australia, the United Kingdom, and the United States. J Med Internet Res. 2020;22(6):e19264.

2. Wosik J, Fudim M, Cameron B, Gellad ZF, Cho A, Phinney D, Tcheng J. Telehealth transformation: COVID-19 and the rise of virtual care. J Am Med Inform Assoc. 2020;27(6):957-62.

3. Lee I, Kovarik C, Tejasvi T, Pizarro M, Lipoff JB. Telehealth: helping your patients and practice survive and thrive during the COVID-19 crisis with rapid quality implementation. J Am Acad Dermatol. 2020;82(5):1213-4. https://doi.org/10.1016/j.jaad. 2020.03.052.
4. Lonergan PE, Washington Iii SL, Branagan L, Gleason N, Pruthi RS, Carroll PR, Odisho AY. Rapid utilization of telehealth in a comprehensive cancer center as a response to COVID-19: crosssectional analysis. J Med Internet Res. 2020;22(7):e19322.

5. Lowery C, DeNicola N. American College of Obstetricians and Gynecologists. Implementing telehealth in practice. Obstet Gynecol. 2020;135(2):e73-9.

6. Dobrusin A, Hawa F, Gladshteyn M, Corsello P, Harlen K, Walsh CX, Gunaratnam NT. Gastroenterologists and patients report high satisfaction rates with telehealth services during the novel coronavirus 2019 pandemic. Clin Gastroenterol Hepatol. 2020;18(11):2393-7.

7. Moran AE, Forouzanfar MH, Roth GA, Mensah GA, Ezzati M, Flaxman A, Naghavi M. The global burden of ischemic heart disease in 1990 and 2010: the Global Burden of Disease 2010 study. Circulation. 2014;129(14):1493-501.

8. Kahn R, Robertson RM, Smith R, Eddy D. The impact of prevention on reducing the burden of cardiovascular disease. Circulation. 2008;118(5):576-85.

9. CDC. "People with Certain Medical Conditions." Centers for Disease Control and Prevention, Centers for Disease Control and Prevention. 2020, https://www.cdc.gov/coronavirus/2019-ncov/ need-extra-precautions/people-with-medical-conditions.html.

10. Adams ML, Katz DL, Grandpre J. Population-based estimates of chronic conditions affecting risk for complications from coronavirus disease. United States Emerging Infectious Diseases. 2020;26(8):1831.

11. Bae S, Kim SR, Kim MN, Shim WJ, Park SM. Impact of cardiovascular disease and risk factors on fatal outcomes in patients with COVID-19 according to age: a systematic review and metaanalysis. Heart. 2021;107(5):373-80.

12. Khera A, Baum SJ, Gluckman TJ, Gulati M, Martin SS, Michos $\mathrm{ED}$, Shapiro MD. Continuity of care and outpatient management for patients with and at high risk for cardiovascular disease during the COVID-19 pandemic: a scientific statement from the American Society for Preventive Cardiology. American Journal of Preventive Cardiology. 2020;1:100009.

13.•• Yuan N, Pevnick JM, Botting PG, Elad Y, Miller SJ, Cheng S, Ebinger JE. Patient Use and Clinical Practice Patterns of Remote Cardiology Clinic Visits in the Era of COVID19. JAMA network open. 2021;4(4):e214157. Findings from this study showed that clinician ordering of diagnostic testing and medications were decreased in telehealth visits. This study also highlighted important barriers that telehealth visits had. This study compared patients with private vs no insurance in terms of their accessibility to telehealth.

14. Krittanawong C. TeleHealth in the digital revolution era. 2021.

15. Poppas A, Rumsfeld JS, Wessler JD. Telehealth is having a moment: will it last?. 2020

16. Singh A, Mountjoy N, McElroy D, Mittal S, Al Hemyari B, Coffey N, Gaines K. Patient perspectives with telehealth visits in cardiology during COVID-19: online patient survey study. JMIR Cardio. 2021;5(1):e25074. This study aimed to determine patient satisfaction and the satisfaction of cardiologists with the new transition to telehealth visits. Through a series of surveys this study showed that cardiologists were able to adapt well to the new transition, however highlighted that the lack of a clinical exam needed to be addressed as that was a persistent drawback brought up by cardiologists surveyed.

17. Lee R. igital divides 2015. Pew Research Center. Published September 22, 2015. Accessed 13 Oct 2020. https://www.pewresearch. org/internet/2015/09/22/digital-divides-2015/

18. Choi N. Relationship between health service use and health information technology use among older adults: analysis of 
the US National Health Interview Survey. J Med Internet Res. 2011;13(2):e33.

19. Hong A, Cho J. Has the digital health divide widened? Trends of health-related internet use among older adults from 2003 to 2011. J Gerontol Ser B Psychol Sci Soc Sci. 2017;72(5):856-63.

20. Gorodeki EZ, Goyal P, Cox ZL, Thibodeau JT, Reay RE, Rasmusson K, Starling RC. Virtual visits for care of patients with heart failure in the era of COVID-19: a statement from the Heart Failure Society of America. J Card Fail. 2020;26(6):448-56.

21. Dang S, Dimmick S, Kelkar G. Evaluating the evidence base for the use of home telehealth remote monitoring in elderly with heart failure. Telemedicine and e-Health. 2009;15(8):783-96.

22. van denBerg MEL, Maeder AJ. Telehealth in the elderly with chronic heart failure: what is the evidence? Telehealth for Our Ageing Society: Selected Papers from Global Telehealth. 2018;2017(246): 18 .

23. Koulaouzidis G, Charisopoulou D. Telemedicine reduces long term mortality in patients with heart failure with reduced ejection fraction. Eur J Prev Cardiol. 28, Issue Supplement_1, May 2021

24. Varma N, Marrouche NF, Aguinaga L, Albert CM, Arbelo E, Choi JI, Varosy PD. HRS/EHRA/APHRS/LAHRS/ACC/AHA worldwide practice update for telehealth and arrhythmia monitoring during and after a pandemic: developed in partnership with and endorsed by the American College of Cardiology (ACC), the American Heart Association (AHA), the Asia Pacific Heart Rhythm Society (APHRS), the European Heart Rhythm
Association (EHRA), the Heart Rhythm Society (HRS), and the Latin American Heart Rhythm Society (LAHRS). EP Europace. 2021;23(2):313.

25. Barrios V, Cosín-Sales J, Bravo M, Escobar C, Gámez JM, Huelmos A, Cequier Á. Telemedicine consultation for the clinical cardiologists in the era of COVID-19: present and future. Consensus document of the Spanish Society of Cardiology. Revista Española de Cardiología (English Edition). 2020;73(11):910-18.

26. Wang L, Nielsen K, Goldberg J, Brown JR, Rumsfeld JS, Steinberg BA, Shah RU. Association of wearable device use with pulse rate and health care use in adults with atrial fibrillation. JAMA Netw Open. 2021;4(5):e215821.

27. Merriel SWD, Andrews V, Salisbury C. Telehealth interventions for primary prevention of cardiovascular disease: a systematic review and meta-analysis. Preventive medicine. 2014;64:88-95.

28. Wosik J, Clowse ME, Overton R, Adagarla B, EconomouZavlanos N, Cavalier J, Pagidipati NJ. Impact of the COVID-19 pandemic on patterns of outpatient cardiovascular care. Am Heart J. 2021;231:1-5.

29. Eurlings CGMJ, Boyne JJ, De Boer RA, Brunner-La Rocca HP. Telemedicine in heart failure-more than nice to have? Neth Heart J. 2019;27(1):5-15.

Publisher's Note Springer Nature remains neutral with regard to jurisdictional claims in published maps and institutional affiliations. 